Proceedings of the Edinburgh Mathematical Society (2008) 51, 297-304 (C)

DOI:10.1017/S0013091506001131 Printed in the United Kingdom

\title{
A NOTE ON RANDOM HOLOMORPHIC ITERATION IN CONVEX DOMAINS
}

\author{
FILIPPO BRACCI \\ Dipartimento di Matematica, Università di Roma 'Tor Vergata', \\ Via della Ricerca Scientifica 1, 00133 Rome, Italy
}

(fbracci@mat.uniroma2.it)

(Received 12 September 2006)

\begin{abstract}
We introduce a geometric condition of Bloch type which guarantees that a subset of a bounded convex domain in several complex variables is degenerate with respect to every iterated function system. Furthermore, we discuss the relations of such a Bloch-type condition with the analogous hyperbolic Lipschitz condition.
\end{abstract}

Keywords: iterated function systems; degenerate subset; complex geodesic; iteration; convex domain

2000 Mathematics subject classification: Primary 32H50

Secondary $70 \mathrm{~K} 99$

\section{Introduction}

Let $D \subset \mathbb{C}^{n}$ be a domain. Let $\left\{f_{j}\right\}$ be a sequence of holomorphic self-maps of $D$. Let $F_{j}:=f_{j} \circ \cdots \circ f_{1}$. The sequence $\left\{F_{j}\right\}$ is called the holomorphic iterated function system (associated with $\left\{f_{j}\right\}$ ). Such systems are encountered naturally in dynamical systems, continued fraction theory and other areas of complex analysis. Given a holomorphic iterated function system, one is interested in knowing its asymptotic behaviour, namely, in knowing the possible limits (in the compact-open topology, for instance) of the sequence. In general such a problem is rather difficult and it suffices to know which conditions guarantee that every limit of $\left\{F_{j}\right\}$ is constant (we refer the reader to $[\mathbf{2}, \mathbf{4}]$ and references therein).

We now examine the problem in more detail. Let $X \subset D$ be a subset of $D$. We say that the set $X$ is degenerate in $D$ if all the limits of any holomorphic iterated function system $\left\{F_{j}\right\}$ for which $f_{j}: D \rightarrow X$ are constant.

In the case when $D=\mathbb{D}:=\{\zeta \in \mathbb{C}:|\zeta|<1\}$, degenerate subdomains have been completely characterized in terms of hyperbolic distance by Beardon et al. $[\mathbf{2}]$ and Keen and Lakic [4]. To state their results, we first introduce some terminology, which will also be needed later.

We denote the Kobayashi distance of $D$ by $k_{D}$ and the corresponding Kobayashi infinitesimal metric by $\kappa_{D}$. For their definition and properties we refer the reader to [5]. 
Here, for the reader's convenience we recall that if $D, D^{\prime} \subset \mathbb{C}^{n}$ are two domains and $f: D \rightarrow D^{\prime}$ is a holomorphic map, then $k_{D^{\prime}}(f(z), f(w)) \leqslant k_{D}(z, w)$ for all $z, w \in D$. Also, if $W \subset D$, then $k_{W}(z, w) \geqslant k_{D}(z, w)$ for all $z, w \in W$ and $\kappa_{W}(z ; v) \geqslant \kappa_{D}(z ; v)$ for all $z \in W$ and $v \in T_{z} W$. Moreover, if $D \subset \mathbb{C}^{n}$ is a convex domain, then $k_{D}: D \times D \rightarrow \mathbb{R}^{+}$ is a (true) distance, continuous on $D \times D$, inducing the Euclidean topology on $D$. In (bounded) convex domains, Lempert's theory $[\mathbf{6}]$ (see also $[\mathbf{1}, \mathbf{5}]$ ) allows us to compute the Kobayashi metric and distance in terms of the so-called Lempert projection device, a crucial tool for our results, not generally available in other types of domain (see below). Note that, for $D=\mathbb{D}, k_{\mathbb{D}}$ is merely the usual Poincaré distance.

Let $X \subset D$. Let us denote by $R(X)$ its Bloch radius, namely

$$
R(X)=\sup \left\{r \geqslant 0: B_{D}(z, r) \subset X\right\},
$$

where $B_{D}(z, r)=\left\{w \in D: k_{D}(w, z)<r\right\}$ is a Kobayashi ball of centre $z \in D$ and radius $r>0$. A subset $X \subset D$ is a Bloch subset of $D$ if $R(X)<+\infty$.

Now let $Z \subseteq D$ be a subdomain of $D$. Then $\kappa_{Z}(z ; v) \geqslant \kappa_{D}(z ; v)$ for all $z \in Z$ and $v \in T_{z} Z$. The hyperbolic Lipschitz constant of $Z$ is defined as

$$
\mu_{D}(Z):=\sup _{z \in Z}\left\{\frac{\kappa_{D}(z ; v)}{\kappa_{Z}(z ; v)}:(z, v) \in T Z, v \neq 0\right\} .
$$

The subdomain $Z$ is called a Lipschitz subdomain of $D$ if $\mu_{D}(Z)<1$.

In $[\mathbf{2}]$ Beardon et al. proved the following result.

Theorem 1.1 (Beardon et al.). Let $U \subset \mathbb{D}$ be a domain. Then

(i) $\tanh \frac{1}{2} R(U) \leqslant \mu_{\mathbb{D}}(U) \leqslant \tanh R(U)$. In particular, $U$ is a Bloch subdomain of $\mathbb{D}$ if and only if it is a Lipschitz subdomain of $\mathbb{D}$,

(ii) if $U$ is a Lipschitz subdomain of $\mathbb{D}$, then $U$ is degenerate in $\mathbb{D}$.

In [4] Keen and Lakic showed that the converse of (ii) also holds, as follows.

Theorem 1.2 (Keen and Lakic). Let $U \subset \mathbb{D}$ be a domain. If $U$ is degenerate in $\mathbb{D}$, then it is a Bloch subdomain of $\mathbb{D}$.

Thus, Theorems 1.1 and 1.2 characterize completely the geometrically degenerate subdomains of $\mathbb{D}$.

In higher dimensions the story is different. In $[\mathbf{2}]$ the following theorem is in fact also proved.

Theorem 1.3. Let $D \subset \subset \mathbb{C}^{n}$ be a domain. If $X \subset D$ is a Lipschitz subdomain of $D$, then $X$ is degenerate in $D$.

However, as Beardon et al. show, Bloch domains are not necessarily Lipschitz domains in several dimensions, and the problem of characterizing in a geometric flavour both Lipschitz and degenerate subdomains of bounded domains in higher dimension remains unsolved.

The aim of the present paper is to present a Bloch-type property that guarantees a subset (not just an open subdomain) of a bounded convex domain to be degenerate. To initiate our definitions and results, we first look at the following example. 
Example 1.4. Let $\left\{g_{j}\right\}$ be any sequence of holomorphic self maps of $\mathbb{D}$ whose associated holomorphic iterated function system has some non-constant limit $g$ and whose image is contained in some set $X^{\prime} \subset \mathbb{D}$. Let us define $f_{j}: \mathbb{B}^{n} \rightarrow \mathbb{B}^{n}$ by $f_{j}\left(z_{1}, \ldots, z_{n}\right)=\left(g_{j}\left(z_{1}\right), 0, \ldots, 0\right)$. Then the holomorphic iterated function system associated with $\left\{f_{j}\right\}$ has its image contained in $X=X^{\prime} \times\{O\}$. The set $X$ is clearly a Bloch subset of $\mathbb{B}^{n}$ because it contains no Kobayashi balls, but the holomorphic iterated function system has a non-constant limit $\left(g\left(z_{1}\right), 0, \ldots, 0\right)$. Note, however, that, by Theorem 1.2, $X \cap(\mathbb{D} \times\{O\})$ cannot be a Bloch subdomain in $\mathbb{D}$ and thus it is not Lipschitz (as a subvariety) of $\mathbb{B}^{n}$ according to Theorem 1.1.

This example suggests that degenerate properties of a subset should be related to Blochness properties of the intersection of that subset with suitably chosen analytic discs. In order to make this argument work (and to choose the right discs), we briefly recall how a Lempert projection device is defined (see [6], [1, Proposition 2.6.22] and [5, Theorem 4.8.12] for further details).

Let $D$ be a bounded convex domain in $\mathbb{C}^{n}$ and let $z_{0} \in D$. Given any point $z \in D$, there exists a complex geodesic $\varphi: \mathbb{D} \rightarrow D$, i.e. a holomorphic isometry between $k_{\mathbb{D}}$ and $k_{D}$, such that $\varphi(0)=z_{0}$ and $\varphi(t)=z$ for some $t \in(0,1)$. A complex geodesic is also an infinitesimal isometry between the Poincare metric and the Kobayashi metric and, given any point $z \in D$ and non-zero direction $v \in T_{z} D$, there exists a complex geodesic containing $z$ and tangent to $v$ at $z$. Note that a complex geodesic is an injective proper map from $\mathbb{D}$ to $D$.

Moreover, for any such a complex geodesic there exists a holomorphic retraction $\rho_{\varphi}$ : $D \rightarrow \varphi(\mathbb{D})$ with affine fibres, i.e. $\rho_{\varphi}$ is a holomorphic self-map of $D$ such that $\rho_{\varphi} \circ \rho_{\varphi}=\rho_{\varphi}$, $\rho_{\varphi}(z)=z$ for any $z \in \varphi(\mathbb{D})$ and $\rho_{\varphi}^{-1}(\varphi(\zeta)) \cap D$ is the intersection of $D$ with a complex hyperplane for all $\zeta \in \mathbb{D}$. We call such a $\rho_{\varphi}$ a Lempert projection associated with $\varphi$. We remark that if $D$ is convex but not strongly convex, then $\rho_{\varphi}$ is not unique in general. For instance, in the bidisc $\mathbb{D} \times \mathbb{D}$ the complex geodesic $\mathbb{D} \ni \zeta \mapsto(\zeta, \zeta)$ has several Lempert projections, such as $\rho^{1}(z, w)=(z, z)$ and $\rho^{2}(z, w)=\left(\frac{1}{2}(z+w), \frac{1}{2}(z+w)\right)$. However, if $D$ is strongly convex, then the Lempert projection (that is, the one with affine fibres) is unique (see [3, Proposition 3.3]).

Furthermore, we let $\tilde{\rho}_{\varphi}:=\varphi^{-1} \circ \rho_{\varphi}: D \rightarrow \mathbb{D}$ and call it the left inverse of $\varphi$, for $\tilde{\rho}_{\varphi} \circ \varphi=\operatorname{id} \mathbb{D}$. The triple $\left(\varphi, \rho_{\varphi}, \tilde{\rho}_{\varphi}\right)$ is a so-called Lempert projection device.

Remark 1.5. For $D=\mathbb{B}^{n}$, the unit ball of $\mathbb{C}^{n}$, the image of the complex geodesic through the points $z \neq w \in \overline{\mathbb{B}}^{n}$ is just the one-dimensional slice

$$
S_{z, w}:=\mathbb{B}^{n} \cap\{z+\zeta(z-w): \zeta \in \mathbb{C}\} .
$$

The Lempert projection is thus given by the orthogonal projection of $\mathbb{B}^{n}$ onto $S_{z, w}$.

Definition 1.6. Let $D \subset \mathbb{C}^{n}$ be a bounded convex domain. We say that a subset $X \subset D$ is 1 -Bloch in $D$ if there exists a $C>0$ such that, for any Lempert projection device $\left(\varphi, \rho_{\varphi}, \tilde{\rho}_{\varphi}\right)$, the subset $\tilde{\rho}_{\varphi}(X)$ is contained in a Bloch subdomain $U_{\varphi}$ of $\mathbb{D}$ with Bloch radius $R\left(U_{\varphi}\right)<C$. 
The main result of this paper is the following.

Theorem 1.7. Let $D \subset \mathbb{C}^{n}$ be a bounded convex domain. Let $X \subset D$ be a subset of $D$ which is 1-Bloch in $D$. Then $X$ is degenerate in $D$.

The proof of Theorem 1.7 is given in $\S 2$. Such a proof does not rely on any Lipschitzian property of 1-Bloch subsets but, as we show in $\S 3$, a 1-Bloch subdomain of $D$ is necessarily Lipschitz in $D$ (so that, in the case when $X$ is a subdomain, Theorem 1.7 also follows from Theorem 1.3). In $\S 3$ we also discuss another natural Blochness condition which is implied by the Lipschitz condition for subdomains, giving some geometric hints on what a Lipschitz subdomain in several complex variables looks like.

\section{Bloch, 1-Bloch and degenerate subsets}

Throughout this section $D$ is a bounded convex domain in $\mathbb{C}^{n}$ and $X \subset D$ denotes a subset of $D$. We begin with the following simple observation.

Proposition 2.1. If $X$ is 1-Bloch, then $X$ is a Bloch subset of $D$.

Proof. Let $r>0$ and let $B_{D}\left(z_{0}, r\right)$ be any Kobayashi ball contained in $X$. Then, for any complex geodesic $\varphi: \mathbb{D} \rightarrow D$ such that $\varphi(0)=z_{0}$, it follows that $\varphi\left(B_{\mathbb{D}}(0, r)\right) \subset$ $B_{D}\left(z_{0}, r\right)$ and therefore $\tilde{\rho}_{\varphi}(\varphi(\mathbb{D}) \cap X)$ contains the hyperbolic disc $B_{\mathbb{D}}(0, r)$. Since $\tilde{\rho}_{\varphi}(\varphi(\mathbb{D}) \cap X) \subset \tilde{\rho}_{\varphi}(X), \tilde{\rho}_{\varphi}(X)$ contains the hyperbolic disc $B_{\mathbb{D}}(0, r)$. Hence, the Bloch radius of any domain in $D$ containing $\tilde{\rho}_{\varphi}(X)$ must be greater than or equal to $r$. Since by the very definition there exists a domain $U_{\varphi} \subset \mathbb{D}$ containing $\tilde{\rho}_{\varphi}(X)$ with Bloch radius less than or equal to $C$, it follows that $r \leqslant C$ and hence $X$ has Bloch radius less than or equal to $C$, and it is a Bloch subset of $D$.

Note that Example 1.4 shows that the converse of Proposition 2.1 is false in general.

In order to prove Theorem 1.7 we need a preliminary fact, which is interesting in itself. First, we recall the following lemma [2, Lemma 3.1]

Lemma 2.2. If $g: \mathbb{D} \rightarrow U$ is holomorphic, then $k_{\mathbb{D}}(g(\zeta), g(\eta)) \leqslant \mu_{\mathbb{D}}(U) k_{\mathbb{D}}(\zeta, \eta)$ for all $\zeta, \eta$ in $\mathbb{D}$.

Then we have the following result.

Proposition 2.3. Let $W_{j} \subset \mathbb{D}$ with $j \in \mathbb{N}$ be Bloch subdomains of $\mathbb{D}$. Assume that there exists $C>0$ such that the Bloch radius $R\left(W_{j}\right)<C$ for all $j \in \mathbb{N}$. If $\left\{g_{j}\right\}$ is a sequence of holomorphic self-maps of $\mathbb{D}$ such that $g_{j}(\mathbb{D}) \subseteq W_{j}$, then the holomorphic iterated function system $\left\{g_{j} \circ \cdots \circ g_{1}\right\}$ has only constant limits.

Proof. By Theorem 1.1 there exists a $c<1$ such that the hyperbolic Lipschitz constant $\mu\left(W_{j}\right)<c$ for all $j$. By Lemma 2.2 it follows that $k_{\mathbb{D}}\left(g_{j}(\zeta), g_{j}(\eta)\right) \leqslant c k_{\mathbb{D}}(\zeta, \eta)$ for all $\zeta, \eta \in \mathbb{D}$ and $j \in \mathbb{N}$. Thus, the sequence $\left\{g_{j}\right\}$ is strictly decreasing with respect to $k_{\mathbb{D}}$ with uniform constant $c<1$ and our statement follows from a contraction-mapping theorem (see [2, Theorem 1.1]).

Now we can prove Theorem 1.7. 
Proof of Theorem 1.7. Let $\left\{f_{j}\right\}$ be a sequence of holomorphic self-maps of $D$ with images contained in $X$. Let $F$ be a limit of the associated holomorphic iterated system $\left\{F_{j}\right\}$ (where $F_{j}:=f_{j} \circ \cdots \circ f_{1}$ ). Up to relabelling, we can assume that $\left\{F_{j}\right\}$ converges to $F$. Assume that $F$ is not constant and that $F(z) \neq F(w)$ for some $z, w \in D$. Let $\varphi_{1}: \mathbb{D} \rightarrow D$ be a complex geodesic such that $\varphi_{1}(0)=z$ and $\varphi_{1}\left(t_{1}\right)=w$ for some $0<t_{1}<1$. Let $\varphi_{j}: \mathbb{D} \rightarrow D, j \geqslant 2$, be a complex geodesic defined by induction as follows:

$$
\varphi_{j}(0)=f_{j-1} \circ \cdots \circ f_{1}(z)=F_{j-1}(z) \quad \text { and } \quad \varphi_{j}\left(t_{j}\right)=f_{j-1} \circ \cdots \circ f_{1}(w)=F_{j-1}(w)
$$

for some $0<t_{j}<1$. Let $g_{j}:=\tilde{\rho}_{\varphi_{j+1}} \circ f_{j} \circ \varphi_{j}$. Then $g_{j}: \mathbb{D} \rightarrow \mathbb{D}$ is holomorphic. By construction, $g_{j} \circ \cdots \circ g_{1}(0)=\tilde{\rho}_{\varphi_{j+1}}\left(F_{j}(z)\right)=0$, while $g_{j} \circ \cdots \circ g_{1}\left(t_{1}\right)=\tilde{\rho}_{\varphi_{j+1}}\left(F_{j}(w)\right)=$ $t_{j+1}$. Now, the family $\left\{\varphi_{j}\right\}$ is a normal family in $D$. Let $\varphi: \mathbb{D} \rightarrow \bar{D}$ be one of its limits. By continuity of the Kobayashi distance it follows that either $\varphi$ is a complex geodesic or $\varphi(\mathbb{D}) \subset \partial D$. Since $\varphi_{j}(0)=F_{j-1}(z) \rightarrow F(z)$ as $j \rightarrow \infty$, it follows that $\varphi$ is in fact a complex geodesic. Moreover, since $\varphi_{j}\left(t_{j}\right)=F_{j-1}(w) \rightarrow F(w)$ as $j \rightarrow \infty$, it follows that there exists $t \in(0,1)$ such that $t_{j} \rightarrow t$ as $j \rightarrow \infty$ and $\varphi(t)=F(w)$. Up to relabelling, we can assume that $\varphi$ is the only limit of $\left\{\varphi_{j}\right\}$.

Next, we claim that the Lempert projections $\left\{\rho_{\varphi_{j}}\right\}$ converge (up to subsequences) in the compact-open topology of $D$ to a Lempert projection $\rho_{\varphi}$ (showing that $\left\{\tilde{\rho}_{\varphi_{j}}\right\}$ converges to $\tilde{\rho}_{\varphi}$ ). To see this, let $\rho$ be any limit of the normal family $\left\{\rho_{\varphi_{j}}\right\}$. Again, up to relabelling, we can assume that $\rho$ is the only limit. First we note that, since $\rho_{\varphi_{j}}\left(\varphi_{j}(\zeta)\right)=\varphi_{j}(\zeta)$ for all $\zeta \in \mathbb{D}$ and $j \in \mathbb{N}$, it follows that $\rho(\varphi(\zeta))=\varphi(\zeta)$ for all $\zeta \in \mathbb{D}$. Thus, in particular, $\rho(D) \subset D$. Now we claim that $\rho(D)=\varphi(\mathbb{D})$. We already know that $\varphi(\mathbb{D}) \subset \rho(D)$. Let $\rho(y) \in \rho(D)$. Then there exists a sequence $\left\{y_{j}\right\} \subset D$ such that $\rho_{\varphi_{j}}\left(y_{j}\right) \rightarrow \rho(y)$ as $j \rightarrow \infty$. Now, $\rho_{\varphi_{j}}\left(y_{j}\right)=\varphi_{j}\left(\zeta_{j}\right)$ for some $\zeta_{j} \in \mathbb{D}$ and we may assume that $\zeta_{j} \rightarrow \zeta \in \overline{\mathbb{D}}$. We claim that $\zeta \in \mathbb{D}$. To see this, let $W_{1} \subset \subset D$ be a relatively compact open neighbourhood of $F(z)$ in $D$ and let $W_{2} \subset \subset D$ be a relatively compact open neighbourhood of $\rho(y)$ in $D$. Since $\varphi_{j}(0) \rightarrow F(z)$ and $\rho_{\varphi_{j}}\left(y_{j}\right) \rightarrow \rho(y)$, we may assume that $\left\{\varphi_{j}(0)\right\} \subset W_{1}$ and $\left\{\rho_{\varphi_{j}}\left(y_{j}\right)\right\} \subset W_{2}$. Let

$$
c:=\sup \left\{k_{D}\left(w_{1}, w_{2}\right): w_{1} \in W_{1}, w_{2} \in W_{2}\right\} .
$$

As $W_{1}$ and $W_{2}$ are relatively compact in $D$ it follows that $c<+\infty$. Now

$$
k_{\mathbb{D}}\left(0, \zeta_{j}\right)=k_{D}\left(\varphi_{j}(0), \varphi_{j}\left(\zeta_{j}\right)\right)=k_{D}\left(\varphi_{j}(0), \rho_{\varphi_{j}}\left(y_{j}\right)\right) \leqslant c .
$$

Hence, $\zeta_{j}$ cannot tend to the boundary of $\mathbb{D}$, for otherwise $k_{\mathbb{D}}\left(0, \zeta_{j}\right) \rightarrow \infty$, which contradicts the previous inequality. Therefore, $\zeta \in \mathbb{D}$. Thus, $\varphi_{j}\left(\zeta_{j}\right) \rightarrow \varphi(\zeta)=\rho(y)$ and hence $\rho(y) \in \varphi(\mathbb{D})$. Finally, since $\rho_{\varphi_{j}} \circ \rho_{\varphi_{j}}=\rho_{\varphi_{j}}$ for any $j$, it follows that $\rho \circ \rho=\rho$. Moreover, it is clear that the fibres of $\rho$ are to be affine, because those of every $\rho_{\varphi_{j}}$ are affine. Hence, $\rho$ is a Lempert projection associated with $\varphi$.

As a result, if $g$ is any limit of the holomorphic iterated function system $\left\{g_{j}\right\}$, it follows that

$$
g(0)=\lim _{j \rightarrow \infty} \tilde{\rho}_{\varphi_{j+1}}\left(F_{j}(z)\right)=\tilde{\rho}_{\varphi}(F(z))=0
$$


and

$$
g(t)=\lim _{j \rightarrow \infty} \tilde{\rho}_{\varphi_{j+1}}\left(F_{j}(w)\right)=\tilde{\rho}_{\varphi}(F(w))=t .
$$

Hence, the holomorphic iterated function system $\left\{g_{j}\right\}$ has a non-constant limit. However, by the very definition of 1-Bloch, $g_{j}: \mathbb{D} \rightarrow W_{j}$ with $W_{j}=\tilde{\rho}_{\varphi_{j+1}}\left(f_{j}\left(\varphi_{j}(\mathbb{D})\right)\right) \subset \tilde{\rho}_{\varphi_{j+1}}(X) \subset$ $U_{j}$, where $U_{j}$ is a Bloch domain in $\mathbb{D}$ with Bloch radius bounded from above by some $C>0$ independent of $j$. This contradicts Proposition 2.3 and we are done.

\section{3. c-Bloch, 1-Bloch and Lipschitz subdomains}

Throughout this section $D$ is a bounded convex domain in $\mathbb{C}^{n}$ and $X \subset D$ denotes a subdomain of $D$.

Definition 3.1. The subdomain $X \subset D$ is $c$-Bloch if there exists a $C>0$ such that for every Lempert projection device $\left(\varphi, \rho_{\varphi}, \tilde{\rho}_{\varphi}\right)$ the (possibly empty) open set $\tilde{\rho}_{\varphi}(X \cap \varphi(\mathbb{D}))$ is contained in a Bloch subdomain of $\mathbb{D}$ with Bloch radius bounded from above by $C$.

By this very definition we have the following result.

Proposition 3.2. A 1-Bloch subdomain $X \subset D$ is c-Bloch.

The converse is, however, false in general, as the following example shows.

Example 3.3. Let $D=\mathbb{B}^{2}$. Let $E\left(e_{1}, R\right)$ denote a horosphere (see, for example, $[\mathbf{1}]$ ) with centre $e_{1}:=(1,0)$, radius $R>0$ and pole $O$; namely

$$
E_{\mathbb{B}^{2}}\left(e_{1}, R\right)=\left\{z \in \mathbb{B}^{2}:\left|1-z_{1}\right|^{2}<R\left(1-\|z\|^{2}\right)\right\} .
$$

Let $X=E_{\mathbb{B}^{2}}\left(e_{1}, 2\right) \backslash E_{\mathbb{B}^{2}}\left(e_{1}, 1\right)$. The domain $X$ is thus formed by the difference of two open complex ellipsoids tangent to each other at the point $(1,0)$. Its closure intersects the boundary of $\mathbb{B}^{2}$ only at the point $(1,0)$. We claim that $X$ is $c$-Bloch but it is not 1-Bloch. Indeed, the orthogonal projection of $X$ on the complex geodesic $\varphi(\zeta)=(\zeta, 0)$ is the horodisc $E_{\mathbb{D}}(1,2)$ of $\mathbb{D}$ with centre 1 and radius 2 which is not a Bloch subdomain of $\mathbb{D}$, showing that $X$ is not 1-Bloch.

To see that $X$ is $c$-Bloch one can argue as follows. If $\varphi: \mathbb{D} \rightarrow D$ is any complex geodesic whose closure contains $(1,0)$, then $\tilde{\rho}_{\varphi}(X \cap \varphi(\mathbb{D}))$ is given by $E_{\mathbb{D}}(1,2) \backslash E_{\mathbb{D}}(1,1)$ (see [1, Proposition 2.7.8(i)]) which is a Bloch subdomain of $\mathbb{D}$ (see $[\mathbf{2}]$ ) with a fixed Bloch radius independent of $\varphi$. As for the other complex geodesics, if $\varphi: \mathbb{D} \rightarrow D$ is any complex geodesic whose closure does not contain $(1,0)$, then $\tilde{\rho}_{\varphi}(X \cap \varphi(\mathbb{D}))$ is given by an annulus $A_{\varphi}$ in $\mathbb{D}$. Such annuli $A_{\varphi}$ stay away from $\partial \mathbb{D}$ independently of $\varphi$, provided that $\varphi$ stays away from a complex geodesic whose closure contains $(1,0)$, while $A_{\varphi}$ 'degenerates' into $E_{\mathbb{D}}(1,2) \backslash E_{\mathbb{D}}(1,1)$ as $\varphi$ tends to a complex geodesic whose closure contains $(1,0)$. Therefore, the Bloch radius of $\tilde{\rho}_{\varphi}(X \cap \varphi(\mathbb{D}))$ is bounded from above independently of $\varphi$. 
We have the following relations among the 1-Bloch, $c$-Bloch and Lipschitz subdomains.

Proposition 3.4. Let $D$ be a bounded convex domain. Let $X \subset D$ be a subdomain.

(i) If $X$ is Lipschitz in $D$, then $X$ is $c$-Bloch in $D$.

(ii) If $X$ is 1-Bloch in $D$, then $X$ is Lipschitz in $D$.

Proof. (i) Let $\varphi: \mathbb{D} \rightarrow D$ be a complex geodesic such that $\varphi(\mathbb{D}) \cap X \neq \emptyset$. The set $U_{\varphi}:=\tilde{\rho}_{\varphi}(X \cap \varphi(\mathbb{D}))$ is a (not necessarily connected) domain in $\mathbb{D}$. Since $\left.\varphi\right|_{U_{\varphi}}: U_{\varphi} \rightarrow X$ is holomorphic, by the monotonicity of the Kobayashi metric we have $\kappa_{X}\left(\varphi(\zeta) ; \mathrm{d} \varphi_{\zeta}(\xi)\right) \leqslant$ $\kappa_{U_{\varphi}}(\zeta ; \xi)$ for all $\zeta \in U_{\varphi}$ and $\xi \in \mathbb{C} \backslash\{0\}=T_{\zeta} U_{\varphi} \backslash\{0\}$. Hence, for all $(\zeta, \xi) \in T U_{\varphi}$ we have

$$
\frac{\kappa_{\mathbb{D}}(\zeta ; \xi)}{\kappa_{U_{\varphi}}(\zeta ; \xi)}=\frac{\kappa_{D}\left(\varphi(\zeta) ; \mathrm{d} \varphi_{\zeta}(\xi)\right)}{\kappa_{U_{\varphi}}(\zeta ; \xi)} \leqslant \frac{\kappa_{D}\left(\varphi(\zeta) ; \mathrm{d} \varphi_{\zeta}(\xi)\right)}{\kappa_{X}\left(\varphi(\zeta) ; \mathrm{d} \varphi_{\zeta}(\xi)\right)} \leqslant c
$$

for some $c<1$ independent of $\varphi$ (because $X$ is Lipschitz in $D$ by hypothesis). Thus, $U_{\varphi}$ is Lipschitz in $\mathbb{D}$ and, by Theorem 1.1 (i), it is a Bloch subdomain of $\mathbb{D}$ with Bloch radius bounded from above by $2 \tanh ^{-1} c$. Hence, $X$ is $c$-Bloch.

(ii) Assume that $X$ is 1-Bloch. This means that there exists a constant $C>0$ such that, for all Lempert projection devices $\left(\varphi, \rho_{\varphi}, \tilde{\rho}_{\varphi}\right)$, the Bloch radius of $\tilde{\rho}_{\varphi}(X)$ is less than or equal to $C$. In particular, by Theorem $1.1(\mathrm{i})$, the open set $\tilde{\rho}_{\varphi}(X)$ is a Lipschitz subdomain of $\mathbb{D}$ with hyperbolic Lipschitz constant bounded from above by $c=\tanh C$.

Fix $z \in X$ and $v \in T_{z} D \backslash\{0\}$. Let $\varphi: \mathbb{D} \rightarrow D$ be a complex geodesic such that $\varphi(0)=z$ and $\mathrm{d} \varphi_{0}(\xi)=v$ for some $\xi \in \mathbb{C}$. Let $\tilde{\rho}_{\varphi}: D \rightarrow \mathbb{D}$ be the left inverse of $\varphi$. By the monotonicity of the Kobayashi metric, considering the holomorphic map $\left.\tilde{\rho}_{\varphi}\right|_{X}: X \rightarrow \tilde{\rho}_{\varphi}(X) \subset \mathbb{D}$ and since $d\left(\tilde{\rho}_{\varphi}\right)_{\varphi(\zeta)} \circ \mathrm{d} \varphi_{\zeta}=\mathrm{id}$, we have

$$
\kappa_{X}(z ; v) \geqslant \kappa_{\tilde{\rho}_{\varphi}(X)}\left(\tilde{\rho}_{\varphi}(z) ; d\left(\tilde{\rho}_{\varphi}\right)_{z}(v)\right)=\kappa_{\tilde{\rho}_{\varphi}(X)}(0 ; \xi) .
$$

Therefore, taking into account the fact that $\kappa_{D}(z ; v)=\kappa_{\mathbb{D}}(0 ; \xi)$, it follows that

$$
\frac{\kappa_{D}(z ; v)}{\kappa_{X}(z ; v)} \leqslant \frac{\kappa_{\mathbb{D}}(0 ; \xi)}{\kappa_{\tilde{\rho}_{\varphi}(X)}(0 ; \xi)} \leqslant \mu_{\mathbb{D}}\left(\tilde{\rho}_{\varphi}(X)\right) \leqslant c
$$

proving that $X$ is Lipschitz in $D$.

Remark 3.5. Proposition 3.4 gives a geometric necessary condition (c-Blochness) for a subdomain to be Lipschitz, and then degenerate. Such a condition is rather easily verified in simple domains such as the unit ball $\mathbb{B}^{n}$ of $\mathbb{C}^{n}$. We do not know whether such a condition is also sufficient; namely, the problem of whether $c$-Blochness implies that the domain is Lipschitz remains unsolved.

Acknowledgements. I thank the referee for precise suggestions and comments which improved the manuscript. 


\section{References}

1. M. ABATE, Iteration theory of holomorphic maps on taut manifolds (Mediterranean Press, Rende, Cosenza, 1989).

2. A. F. Beardon, T. K. Carne, D. Minda and T. W. NG, Random iteration of analytic maps, Ergod. Theory Dynam. Syst. 24 (2004), 659-675.

3. F. Bracci, G. PAtrizio and S. Trapani, The pluricomplex Poisson kernel for strongly convex domains, Trans. Am. Math. Soc., in press.

4. L. KEEN AND N. LAKIC, Random holomorphic iterations and degenerate subdomains of the unit disk, Proc. Am. Math. Soc. 134 (2005), 371-378.

5. S. Kobayashi, Hyperbolic complex spaces, Grundlehren der mathematischen Wissenschaften, Volume 318 (Springer, 1998).

6. L. LEMPERT, La métrique de Kobayashi et la representation des domaines sur la boule, Bull. Soc. Math. France 109 (1981), 427-474. 